\title{
O pré-natal como estratégia de prevenção a violência obstétrica
}

\author{
Prenatal care as a strategy to prevent obstetric violence
}

\section{La atención prenatal como estrategia para prevenir la violencia obstétrica}

Nataly Yuri Costa1*, Laíze Rúbia Silva Corrêa ${ }^{1}$, Gabriela Xavier Pantoja ${ }^{1}$, Armando Sequeira Penela ${ }^{1}$, Sávio Felipe Dias Santos ${ }^{1}$, Isadora Menezes Franco ${ }^{1}$, Nicole de Oliveira Araujo', Veronica Vale de Barros ${ }^{1}$, Paula Valéria Dias Pena Costa ${ }^{1}$, Laura Maria Vidal Nogueira ${ }^{1}$.

\section{RESUMO}

Objetivo: Descrever a educação em saúde realizada durante pré-natal para prevenção da violência obstétrica. Relato de experiência: A partir do atendimento do pré-natal e observação da realidade do local de prática, as discentes perceberam o déficit de conhecimento das gestantes a respeito do tema violência obstétrico, planejando, assim, um retorno à realidade por meio de metodologia ativa, a qual dividiu-se em dois momentos: o primeiro durante as consultas de pré-natal seguintes, havendo a discussão do tema com as gestantes; e uma palestra ministrada pelas discentes abordando o conceito, tipos de violência, direitos da gestante, e como denunciar a violência obstétrica. Ao final, foi realizada uma atividade lúdica denominada "o corredor do cuidado", proporcionando um momento de reflexão e empoderamento para as gestantes presentes, bem como favorecer a ligação entre o binômio mãe/filho. Considerações finais: A experiência proporcionou as acadêmicas maior visibilidade e consciência da importância do tema, evidenciando a necessidade de fortalecer a discussão sobre a violência obstétrica com as gestantes durante o pré-natal, orientando-as desde o princípio da gravidez, em prol do resguardo à saúde da mãe e filho.

Palavras-chave: Violência contra a mulher, Enfermagem obstétrica, Cuidado pré-natal.

\section{ABSTRACT}

Objective: To describe health education carried out during prenatal care to prevent obstetric violence. Experience report: From the prenatal care and observation of the reality of the place of practice, the students realized the lack of knowledge of pregnant women regarding the obstetric violence theme, thus planning a return to reality through an active methodology, which was divided into two moments: the first during the following prenatal consultations, with the topic being discussed with the pregnant women; and a lecture given by the students addressing the concept, types of violence, rights of the pregnant woman, and how to report obstetric violence. At the end, a playful activity called "the care corridor" was carried out, providing a moment of reflection and empowerment for the pregnant women present, as well as favoring the connection between the mother / child binomial. Final considerations: The experience provided the students with greater visibility and awareness of the importance of the topic, highlighting the need to strengthen the discussion on obstetric violence with pregnant women during prenatal care, guiding them from the beginning of pregnancy, in favor of safeguarding the health of women. mother and son.

Keywords: Violence against women, Obstetric nursing, Prenatal care.

\section{RESUMEN}

Objetivo: Describir la educación en salud realizada durante la atención prenatal para prevenir la violencia obstétrica. Informe de experiencia: A partir del cuidado prenatal y la observación de la realidad del lugar de práctica, los estudiantes se dieron cuenta de la falta de conocimiento de las mujeres embarazadas sobre el tema de la violencia obstétrica, por lo que planearon un retorno a la realidad a través de una metodología activa. , que se dividió en dos momentos: el primero durante las siguientes consultas prenatales, con el tema discutido con las mujeres embarazadas; y una conferencia impartida por los estudiantes sobre el concepto, los tipos de violencia, los derechos de la mujer embarazada y cómo denunciar la violencia obstétrica. Al final, se llevó a cabo una actividad lúdica llamada "corredor de atención", que proporcionó un momento de reflexión y empoderamiento para las mujeres embarazadas presentes, además de favorecer la conexión entre el binomio madre / hijo. Consideraciones finales: La experiencia proporcionó a los estudiantes una mayor visibilidad y conciencia de la importancia del tema, destacando la necesidad de fortalecer la discusión sobre la violencia obstétrica con mujeres embarazadas durante la atención prenatal, guiándolas desde el comienzo del embarazo, a favor de salvaguardar la salud de las mujeres. madre e hijo.

Palabras clave: Violencia contra la mujer, Enfermería obstétrica, Atención prenatal.

${ }^{1}$ Universidade do Estado do Pará (UEPA), Belém - PA. *E-mail: natalyyuricosta@gmail.com

SUBMETIDO EM: $8 / 2020$

ACEITO EM: 9/2020

PUBLICADO EM: 12/2020 


\section{INTRODUÇÃO}

A gestação é um processo marcado por mudanças físicas, fisiológicas e psicológicas, que produzem dúvidas, vulnerabilidades à saúde da mulher e expectativas sobre o ciclo gravídico, o parto e o puerpério. Nesse sentido, o acompanhamento pré-natal é de suma importância para o desenvolvimento de ações de promoção à saúde, prevenção de doenças e assistência à saúde da gestante, com o intuito de assegurar o desenvolvimento saudável da gravidez, de auxiliar a mãe e a família no processo de mudança e adaptação, bem como de orientar a respeito dos direitos da gestante e da puérpera (ARAÚJO SM, et al., 2013; BRASIL, 2000).

As Unidades Básicas de Saúde (UBS) e Estratégia Saúde da Família (ESF), por meio da consulta prénatal, são pontos de acesso preferencial da gestante ao sistema de saúde, onde essas consultas são realizadas mensalmente e de forma intercaladas pelo profissional médico e enfermeiro (BRASIL, 2012; DUARTE SJH e DE ANDRADE SMO, 2006).

Assim, é de competência do profissional enfermeiro, segundo a Lei do Exercício Profissional, acompanhar o pré-natal de baixo risco, requisitar exames, prescrever medicamentos estabelecidos em programas de saúde pública e em rotina aprovada pela instituição de saúde e, principalmente, repassar conhecimentos que priorizem a vida da mãe e da criança (CUNHA MA, et al., 2009; ARAÚJO SM, et al., 2010).

O enfermeiro possui embasamento técnico-científico e respaldo legal para oferecer assistência de qualidade e humanizada à mulher gestante, fornecendo orientações sobre o seu direito a atendimento digno, no decorrer da gestação, parto e puerpério.

Essas orientações contribuem para redução da morbimortalidade e diminuição dos casos de Violência Obstétrica (VO), a partir das boas práticas assistenciais e de humanização que diminuem as dores e tempo de parto, priorizando o processo natural, evitando assim possível trauma (BRASIL, 1986).

Sendo assim, o pré-natal enquanto estratégia de prevenção a VO e resguardo da saúde do binômio mãe/filho, torna-se um espaço fundamental para a realização de atividades educativas que objetivam prevenir a VO verbal, física ou psicológica, a exemplo da manobra de Kristeller, episiotomia de rotina ou sem indicação, privação da presença do acompanhante e de escolha da posição de parto (SENA LM e TESSER CD, 2016; DIAS RL, et al., 2015).

Resultados de estudos ratificam a ocorrência da VO por ocasião da parição, com os seguintes achados: $47,6 \%$ das mulheres inclusas em uma pesquisa feita no Sudeste da Bahia foram sujeitas ao puxo respiratório prolongado e dirigido e $42,9 \%$ foram submetidas a tração de cordão umbilical para agilizar a dequitação, portanto, práticas reconhecidas como VO.

Em outro estudo, $25 \%$ de mulheres informaram sofrer violência durante o parto, sendo $74 \%$ em hospitais públicos, o que corrobora a necessidade da educação em saúde para informar as gestantes durante o prénatal visando a prevenção de tais práticas (RODRIGUES RL e DAS MERCES MC, 2017; PAZ A, et al., 2018).

A educação em saúde realizada durante o acompanhamento pré-natal possibilita o esclarecimento de dúvidas, troca de vivências, criação de vínculo e diálogo entre o profissional enfermeiro, a gestante e sua família, gerando aprendizado crítico para enfrentamento das adversidades, além de sedimentar os direitos e deveres como cidadãs (LIMA VKS, et al., 2019; BLANK EB, et al., 2019).

Nesse sentido, estudo realizado com 555 gestantes que participaram de ação educativa sobre VO, no formato de exposição, apontou aumento significativo no conhecimento dessas mulheres acerca da temática após a participação. Foi constatado que antes da visita à exposição, apenas $42,0 \%$ detinha um bom/muito bom conhecimento sobre $\mathrm{VO}$ e, após a exposição, a proporção passou para $91,2 \%$.

Este resultado, demonstra a importância da realização de educação em saúde a respeito do tema para promoção da saúde do binômio mãe/filho, prevenção de práticas violentas e redução da morbimortalidade materna e neonatal (LANSKY S, et al., 2019). A partir do exposto, estabeleceu-se como objetivo descrever a educação em saúde realizada durante o pré-natal para prevenção da violência obstétrica. 


\section{RELATO DE EXPERIÊNCIA}

Trata-se de um relato de experiência vivenciado por acadêmicas de enfermagem de uma universidade pública no Estado do Pará, caracterizada como ação de educação em saúde com o intuito de orientar gestantes sobre a prevenção da VO, por ocasião do pré-parto, parto e puerpério. A estratégia educativa ocorreu por meio das consultas de pré-natal, ofertado pelo Sistema Único de Saúde (SUS), em um Centro de Saúde, localizado na capital de Belém do Pará.

A ação educativa foi planejada e executada segundo a metodologia do Arco de Maguerez, dividida em seis etapas: observação da realidade, realizada por meio de uma visita ao local escolhido como campo de pesquisa; levantamento dos pontos-chave elencados como problemas em potenciais, possíveis de identificação a partir da observação; teorização, efetivada a partir da fundamentação teórica extraída das bases de dados; hipóteses de solução, que analisaram as propostas de intervenção para minimizar ou solucionar o problema identificado; e por fim, retorno à realidade, com uma intervenção no grupo de gestantes com o objetivo de promover a ação educativa (BERBEL NAN, 2011).

A observação da realidade ocorreu durante a prática do componente curricular Enfermagem em Saúde da Mulher na Atenção Primária, entre os meses de agosto e setembro de 2019, a partir do acompanhamento da rotina das consultas de pré-natal, identificando pontos-chave fundamentais para consolidação do estudo, já que em meio à consulta, com o enfermeiro da unidade, os acadêmicos puderam fazer questionamentos básicos sobre o conceito, formas de ocorrência e indivíduos que praticavam a VO, levando-nos a constatação que a maioria das gestantes tinham pouco conhecimento sobre o tema.

A etapa seguinte, que consistiu na teorização, foi realizada na Biblioteca Virtual em Saúde (BVS), e as bases de dados escolhidas foram Literatura Latino-Americana e do Caribe em Ciências da Saúde (LILACS) e Scientific Electronic Library Online (SciELO), a fim de identificar evidências científicas relacionadas ao tema para embasamento da análise posterior.

Este levantamento subsidiou a elaboração de hipóteses de solução para orientação das gestantes, que se deu com retorno à realidade valendo-se de metodologia ativa, que inicialmente permitiu fazer levantamento a respeito do conhecimento sobre VO.

A partir de então, foram estabelecidos dois momentos de intervenção na realidade: i) construção de um espaço dialogado, com duração de 15 a 20 minutos, sobre VO conduzido pelas acadêmicas de enfermagem, durante as consultas de pré-natal das gestantes matriculadas na UBS; ii) no segundo momento foi desenvolvida ação educativa por meio de uma dinâmica chamada "corredor do cuidado", utilizando recursos visuais, e distribuição de folders educativos, abordando o conceito, a identificação dos tipos de VO, instituições e profissionais, possivelmente envolvidos, e a legislação que garante o amparo legal, além de orientação para denúncia.

O desenvolvimento do primeiro momento, durante a consulta do pré-natal, possibilitou identificar no grupo que algumas multigestas reconheceram que haviam sido vítimas de VO. Muitas dúvidas foram verbalizadas em relação ao direito à presença do acompanhante no momento do parto, pois era corriqueiro em gestações anteriores ficarem sozinhas com os profissionais de saúde. Ademais, demonstraram desconhecimento quanto os canais para denunciar tais atos.

No segundo momento, já na ação educativa, foi feita explanação a respeito da VO, inclusive dados estatísticos, com auxílio de um vídeo ilustrativo contendo relato de mulheres que sofreram VO e como esse trauma perdurava em suas vidas. A ação permitiu identificar que além do conhecimento agregado, a ação propiciou, às gestantes, maior visibilidade acerca do assunto e, corroborou a importância de se expor a gestante a essa realidade, para evitar sofrimentos momentâneos e traumas para o resto da vida à mãe e a criança.

Por fim, com objetivo de desenvolver autoconfiança, empoderamento e o autocuidado, as discentes realizaram, com as gestantes, uma dinâmica intitulada "corredor do cuidado". Para tanto, iniciaram com uma atividade de musicoterapia para relaxamento corporal e mental. Após, foram dispostas duas fileiras: uma formada pelas acadêmicas e outra pelas gestantes, formando o "corredor". 
Com a movimentação orientada uma gestante passava no meio do "corredor" e caminhava vagarosamente, de olhos fechados, escutando, de cada integrante, palavras de cuidado, de carinho, de encorajamento, de força, de empoderamento e segurança, com o objetivo de fortalecer a autoestima dessas mulheres, sobretudo, das gestantes adolescentes, pois era perceptível a insegurança e o medo diante de uma gravidez, essencialmente pela ausência da figura paterna.

Ao final da ação as participantes mostraram-se satisfeitas e acolhidas relataram que gostariam que momentos como esse fizessem parte da rotina de pré-natal de toda e qualquer Unidade de Saúde, para que outras mulheres pudessem ter suas dúvidas esclarecidas de forma lúdica e acessível.

A dinâmica "corredor do cuidado" também cumpriu seu objetivo ao instigar o sentimento de sororidade e empoderamento entre as mulheres, perpassando barreiras impostas pelo medo e pela insegurança, sentimentos muito comuns durante a gestação.

\section{DISCUSSÃO}

O conhecimento compartilhado a gestante durante a consulta do pré-natal é essencial no período gravídico, parto e pós-parto. É importante que a gestante se sinta acolhida, segura, escutada pelo enfermeiro; e seja orientada sobre os processos fisiológicos que ocorrem durante o pré-parto, e os procedimentos a serem realizados durante o parto e pós-parto (ASSUNÇÃO CS, et al., 2019).

Atualmente, muitas parturientes desconhecem atos, omissões ou condutas inadequadas realizadas por profissionais de saúde durante o pré-parto, parto e pós-parto, que são reconhecidas como VO, acarretando sofrimento, dor e angústia à mulher, que naturaliza essa violência, pois não tem conhecimento de seus direitos enquanto gestante (DA SILVA WB, et al., 2019).

A educação em saúde desenvolvida durante o pré-natal, não somente favorece a gestante com informações sobre a gravidez, parto e pós-parto, como também estimula e incentiva a autonomia da mulher, fortalecendo sua autoconfiança, evidenciando seu protagonismo diante da gravidez e poder de escolha frente aos procedimentos realizados com seu corpo (DE ANDRADE LFB, et al., 2017).

Assim, diante das práticas violentas ainda presentes nos serviços de saúde, como Manobra Kristeller, violência verbal, episiotomia de rotina sem indicação, uso inadequado de ocitocina e exame de toque vaginal repetitivo, é essencial que o enfermeiro, por ocasião da consulta, oriente a gestante acerca dos seus direitos e práticas consideradas VO, realizadas sem seu consentimento (OLIVEIRA MC e DAS MERCÊS MC, 2017; DO NASCIMENTO LC, et al., 2017).

Durante o trabalho de parto, parto e pós parto é fundamental que as decisões da gestante sejam respeitadas e ouvidas pela equipe, promovendo autonomia e segurança a mulher, somado ao acolhimento e assistência humanizada à mãe e ao bebê, esclarecendo dúvidas, favorecendo para que esse processo seja com o mínimo de dor, seja física ou psicológica (SANTOS ALM, DE SOUZA MHT, 2017).

Um estudo feito no estado de Tocantins com gestantes evidenciou a VO sofrida devido ao despreparo dos profissionais de saúde no processo assistencial, somado a falta de informação por parte da mãe e do acompanhante (GUIMARÃES LBE, et al., 2018).

Orientar a gestante sobre seus direitos, sobretudo, sobre o direito a presença do acompanhante, seja o companheiro ou membro da família durante o parto é fundamental para que a mulher se sinta segura. Com isso, é necessário que o acompanhante esteja presente nas consultas de pré-natal e seja orientado pelo profissional de enfermagem sobre a VO, compreendendo sua ocorrência, como prevenir e denunciar (DINIZ CSG, et al., 2014).

É assegurado pela Lei $n^{0} 11.108$ de 7 de abril de 2005 o direito ao acompanhante durante o trabalho de parto, parto e pós parto imediato, no âmbito do SUS, o que promove segurança, conforto, suporte emocional, aumentando o número de partos vaginais espontâneos e diminuindo a analgesia intraparto (BRASIL, 2005).

O pré-natal é uma ferramenta de aprendizado, de trocas de conhecimentos, experiências, em prol do melhor desenvolvimento do bebê e saúde da mãe. Assim, realizar educação em saúde a fim de prevenir a VO torna-se fundamental na diminuição dessa prática, empoderando a mulher acerca de seus direitos, preservando sua autonomia e fortalecendo sua autoconfiança frente à gravidez. 
Diante desse quadro, deve se enfatizar o papel do enfermeiro como profissional capacitado a realizar ações educativas que promovam a saúde do binômio mãe/ filho, a partir de palestras, campanhas, rodas de conversa, entre outros (SILVA CS, et al., 2016; DA SILVA WB, et al., 2019).

As atividades realizadas permitiram que as acadêmicas visualizassem a relevância de repassar as informações sobre a VO a gestante desde o início da gestação, tendo o pré-natal como um meio de orientação à mulher que desconhece tais práticas violentas e abusivas que ocorrem.

A metodologia ativa utilizada foi fundamental para evidenciar as lacunas de conhecimento sobre a VO, desde de a percepção do problema, até a intervenção para amenizá-lo, visualizando a educação em saúde como principal estratégia de prevenção a VO.

É necessário que o profissional de enfermagem esteja preparado com o conhecimento cientifico, epidemiológico e prático sobre o tema, criando um vínculo e empoderando essa parturiente sobre seus direitos, além de orientar o acompanhante sobre as formas de ocorrência da violência obstétrica e a importância da sua presença em toda gestação, repassando o sentimento de segurança à mãe, tornando a gestação um processo humanizado e acolhedor.

\section{REFERÊNCIAS}

1. ARAÚJO SM, et al. A importância do pré-natal e a assistência de enfermagem. VEREDAS FAVIP - Revista Eletrônica de Ciências, 2013; 3(2): 61-67.

2. ASSUNÇÃO CS, et al. O Enfermeiro no Pré-Natal: Expectativas de Gestantes. Revista de Pesquisa: Cuidado é Fundamental Online, 2019; 11(3):576-581.

3. BERBEL NAN. As metodologias ativas e a promoção da autonomia de estudantes. Semina: Ciências Sociais e Humanas, 2011; 32(1): 25-40.

4. BLANK EB, et al. Práticas educativas para (re)significar o parto e o nascimento no olhar de puérperas. SALUSVITA, Bauru, 2019; 38 (3): 581-595.

5. BRASIL. Ministério da Saúde. Secretaria de Atenção à Saúde. Atenção ao pré-natal de baixo risco. Cadernos de Atenção n. 32. Brasília: Ministério da Saúde; 2012.

6. BRASIL. Ministério da Saúde. Gabinete do Ministro. Portaria no 569, de 1ํ de junho de 2000. Estabelece princípios e diretrizes para a estruturação do Programa de Humanização no Pré-natal e Nascimento. Diário Oficial da União, 2000.

7. BRASIL. Ministério da Saúde. Lei no 11.108 de 07 de abril de 2005. Altera a Lei no 8.080, de 19 de setembro de 1990, para garantir às parturientes o direito à presença de acompanhante durante o trabalho de parto, parto e pós-parto imediato, no Âmbito do Sistema Único de Saúde - SUS. Diário Oficial da União, 2005.

8. BRASIL. Lei $n^{\circ} 7.498 / 86$ de 25 de junho 1986. Dispõe sobre a regulamentação do exercício da Enfermagem e outras providências. Brasília (DF); 1986.

9. CUNHA MA, et al. Assistência pré-natal: competências essenciais desempenhadas por enfermeiros. Escola Anna Nery Revista de Enfermagem, 2009; 13(1): 145-153.

10. DA SILVA WB, et al. Educação em saúde acerca da prevenção da violência obstétrica: relato de experiência, Campinas. Revista Eletrônica Acervo em Saúde, 2019; 11(14): e1163.

11. DE ANDRADE LFB, et al. Boas Práticas na atenção obstétrica e sua interface com a humanização da assistência, Rio de Janeiro. Revista de enfermagem UERJ, 2017; 25 (e26442); 1-7.

12. DIAS RL, et al. Violência obstétrica: perspectiva da enfermagem. Revista Rede de Cuidados em Saúde, 2015; 9(2):1-4.

13. DINIZ CSG, et al. Implementação da presença de acompanhantes durante a internação para o parto: dados da pesquisa nacional Nascer no Brasil. Caderno de Saúde Pública, 2014; 30 (Supl 1):140-153.

14. DO NASCIMENTO LC, et al. Relato de puérperas acerca da VO nos serviços públicos, Recife. Revista de enfermagem UFPE online, 2017;11 (5); 2014-2023.

15. DO PRADO ML, et al. Arco de Charles Maguerez: refletindo estratégias de metodologia ativa na formação de profissionais de saúde. Escola Anna Nery Revista de Enfermagem, 2012; 16(1): 172-177.

16. DUARTE SJH, DE ANDRADE SMO. Assistência pré-natal no Programa Saúde da Família. Escola Anna Nery Revista de Enfermagem, 2006; 10(1): 121-125.

17. GUIMARÃES LBE, et al. Violência obstétrica em maternidades públicas do estado do Tocantins. Revista Estudos Feministas, 2018; 26(1): 1-11.

18. LANSKY S, et al. Violência obstétrica: influência da Exposição Sentidos do Nascer na vivência das gestantes. Ciência \& Saúde Coletiva, 2019; 24(8): 2811-2823.

19. LIMA VKS, et al. Educação em saúde para gestantes: a busca pelo empoderamento materno no ciclo gravídico-puerperal. Revista de Pesquisa: Cuidado é Fundamental Online, 2019; 11(4): 968-975.

20. OLIVEIRA MC, DAS MERCES MC. Percepções sobre violências obstétricas na ótica de puérperas. Revista de enfermagem UFPE online, 2017; 11 (6), 2483-2489, 2017.

21. PAZ A, et al. Violência obstétrica no Brasil: Uma Revisão Bibliográfica. Centro Universitário São Camilo, 2018; 4(1):257-270.

22. RODRIGUES RL, DAS MERCES MC. Prevalência de violências obstétricas em um município do sudoeste da Bahia: um estudo piloto. Enfermagem Brasil, 2017; 16(4): 210-7.

23. SANTOS ALM, DE SOUZA MHT. Elaboração de novas tecnologias em enfermagem: utilização de uma cartilha para prevenção. Revista de enfermagem UFPE online, Recife, 2017; 11(10):3893-3898.

24. SENA LM, TESSER CD. Violência obstétrica no Brasil e o ciberativismo de mulheres mães: relato de duas experiências. Interface-Comunicação, Saúde, Educação, 2016; 21(60): 209-220.

25. SILVA CS, et al. Atuação do enfermeiro na consulta pré-natal: limites e potencialidades. Revista Online de Pesquisa Cuidado é Fundamental, 2016; 8(2):4087- 4098. 